\title{
On the Analysis of Unreliable Markovian Multiserver Queue with Retrials and Impatience
}

\author{
Meriem Elhaddad and Faiza Limam-Belarbi*
}

\begin{abstract}
This paper concerns an approximate analysis of a Markovian multiserver infinite source retrial queuing with impatience, in which all the servers are subject to breakdown and repairs. Customer who find the total number of busy and failed servers equal to $s$,i.e, he is given to choice to enter a retrial orbit for an random amount of time before attempting to reccess an available server or enter the queue of size $q$. Customer waiting in the queue start being served as an idle or repaired server assigned to them, they can also leave the queue and enter orbit due to impatience. Customers whose service is interrupted by a failure may have the option of leaving the system entirely or returning to the orbit to repeat or resume service. We assume that each server has its own dedicated repair person, and repairs begin immediately following a failure and all process are assumed to be mutually independent.

The simultaneous effect of customer balking, impatience and retrials is analyzed. We try to approximate the steady-state joint distribution of the number of customers in orbit and the number of customers in the service area using a phase-merging Algorithm.
\end{abstract}

Keywords: Retrial queue; Multi server; Impatient; Breakdown and repair of service; Phase merging algorithm

AMS Subject Classification (2010): 60K25,90B22, 68M20.

${ }^{*}$ Corresponding author

\section{Introduction}

Queuing systems in which arriving customers who find all servers and waiting positions occupied may retry for service after a period of time is called Retrial Queues. Retrial queue are devoted by large literature, his study is motivated by the advent of new technologies including applications of switching systems Phones and also to computer and telecommunication net-works. One of the earliest papers in retrial queue was "On the influence of repeated calls in the theory of probabilities of blocking" by Kosten [16]. In 1957, Cohen [7] published "Basic problem of telephone traffic theory and the influence of repeated calls in which he considered the more general $M / M / c$ retrial queue with impatient customers. Aguir and al. [1] and Altalejo and al. [4] modeled a call center as the $M / M / m$ retrial queue with exponential impatience time. Shin and Choo [17] considered an $M / M / s$ retrial queue with balking and reneging in which authors assumed that probabilities that the balking customers and reneging customers join orbit may depend on the number of customer in service facility. For detailed description of $M / M / s$ queue with impatient retrial see Shin [17].

Due to the complexity of the analytical results obtained during the analysis of call-back queue systems, various authors have tried to develop numerical and approximate methods in order to be able to analyze these systems with repeated calls. Aguir and al. [1], estimate the total arrival rate, the sum of external arrival rate and retrial rate for an $M / M / s$ retrial queue with balking and reneging by using fluid flow approxmation. Subramanian and al. [18], bublished " $M / M / s$ retrial queuing system with breakdown and repair of services" by using Direct Truncation Method. Falin [11] consider two approximations in order to study the system behavior for low retrial intensity.

For detailed survey of retrial queues and bibliographical information see Falin [10], Artalejo [2], [3], [5], monograph by Falin and Templeton [12], Gomez-Corral [4], Melikov [14] and Elhaddad [9].

Received : 20-02-2018, Accepted : 07-10-2019 
In this paper, we applied an approximate method in order to investigate an unreliable $M / M / s$ retrial queues with failed servers and discuss the influence of customer balking and impatience as well as the effect of the service area's on the mean orbit length using a phase-merging algorithm due to Korolyuk [13] and Courtois [8]. These kinds of models are readily available in real life situations, for example, consider the transmission of messages in facsimile "net-works" having the autorepeat facilities. If transmission is not successfully completed for some reasons such as a power failure or a transmission error, then the message leaves the server and joins the buffer with some probability and leaves the system with the complimentary probability.

Our paper is organized as follows: In Section 2, we provide the formal model description and state the assumptions that are needed to implement the approximation procedure. In Sections 3 and 4, the algorithm is formally reviewed for $q=1$ and $s=1$. In Section 5, we derive approximations for the steady-state probabilities and several standard queueing performance measures. In Section 6, we applied the Algorithm for a higher values of $s$ and $q$ and in Section 7, based on the results obtained in section 5 for the case $s=1$ and $q=1$ we shall present some numerical example by varying the values of parameters model, which are chosen to satisfy the stationary condition given in Section 6, to illustrate how the various parameters of the model influence on the mean orbit length.

\section{Model Description}

The unreliable homogeneous multiservers infinite source retrial queuing system considered is described as follows: There are an infinite of sources each of which generates customers according to a Poisson process with rate $\lambda(\lambda>0)$. The customer who finds one of the servers idle and not failed occupies it immediately. However, if he finds the total number of busy and failed servers equal to $s$ may balk, i.e., he is given the choice to enter the retrial orbit with probability $f e^{-}\left(0 \leq f e^{-} \leq 1\right)$ or enter the queue of size $q$ with probability $f_{e}^{+}=1-f_{e}^{-}$. Let $c$ be the finite capacity of the customer in service area $(c=s+q)$. Customers located in the orbit wait for an exponentially distributed time and retry to enter the service area with retrial rate $\nu(\nu>0)$. Busy Server complete requests with service rate $\mu$ and customers leave the system. Failures for the $s$ servers occur via a Poisson process with rate $\xi(\xi>0)$ and the repair times for each server are exponentially distributed with rate $\alpha(\alpha>0)$.

Customers waiting in the queue start being served as soon as an idle or repaired server assigned to them, they can also leave the queue and enter orbit with rate $\eta$ due to impatience.

The customer whose service is interrupted by a failure may enter the retrial orbit with probability $q_{f}\left(0 \leq q_{f} \leq 1\right)$ or abandons the service with probability $1-q_{f}$. Customers are lost if they decide not to join the orbit.

All times involved in the model are assumed to be mutually independent of each other.

\section{Underlying Markov Chain}

The system state of our model as described in Section 2 can be represented by a trivariate stochastic process, $\{(N(t), C(t), F(t)): t \geq 0\}$, where $N(t)$ is the random variable which represents the number of customers in the orbit at time $t, C(t)$ is the random variable which represents the number of customers in the service area at time $t$ and $F(t)$ represents the number of failed servers at time $t$.

Since all the random times are exponentially distributed, the stochastic process is a continuous-time Markov chain (CTMC) on the state space $A=\{(i, j, k): i \geq 0 ; j \in\{0,1,2, \ldots q+s\} ; k \in\{0,1,2, \ldots s\}\}$. We assume that as $t \rightarrow \infty$ the steady-state distribution of $\{(N(t), C(t), F(t)): t \geq 0\}$ exists.

Define $p(i, j, k)$ as the limiting probability that the system is in the state $(i, j, k)$ where $(i, j, k) \in A$. Defined mathematically

$$
p(i, j, k)=\lim _{t \rightarrow \infty} P(N(t)=i, C(t)=j, F(t)=k) .
$$

In the following, we restrict our study to the case $s=1$ and $q=1$, the state space $A=\{(i, j, k): i \geq 0$; $j \in\{0,1,2\} ; k \in\{0,1\}\}$. The state transition diagram of the corresponding CTMC is shown in Figure 1 . The levels correspond directly to the size of the orbit and the pairs represent the number of customers in service area and the number of failed servers, respectively.

The statistical equilibrium equations of the system can be describe as follows: for $i=0$

and for $i \geq 1$

$$
(\lambda+\xi) p(0,0,0)=\mu p(0,1,0)+\alpha p(0,1,1)
$$

$$
(\lambda+\xi+i \nu) p(i, 0,0)=\mu p(i, 1,0)+\alpha p(i, 1,1)
$$




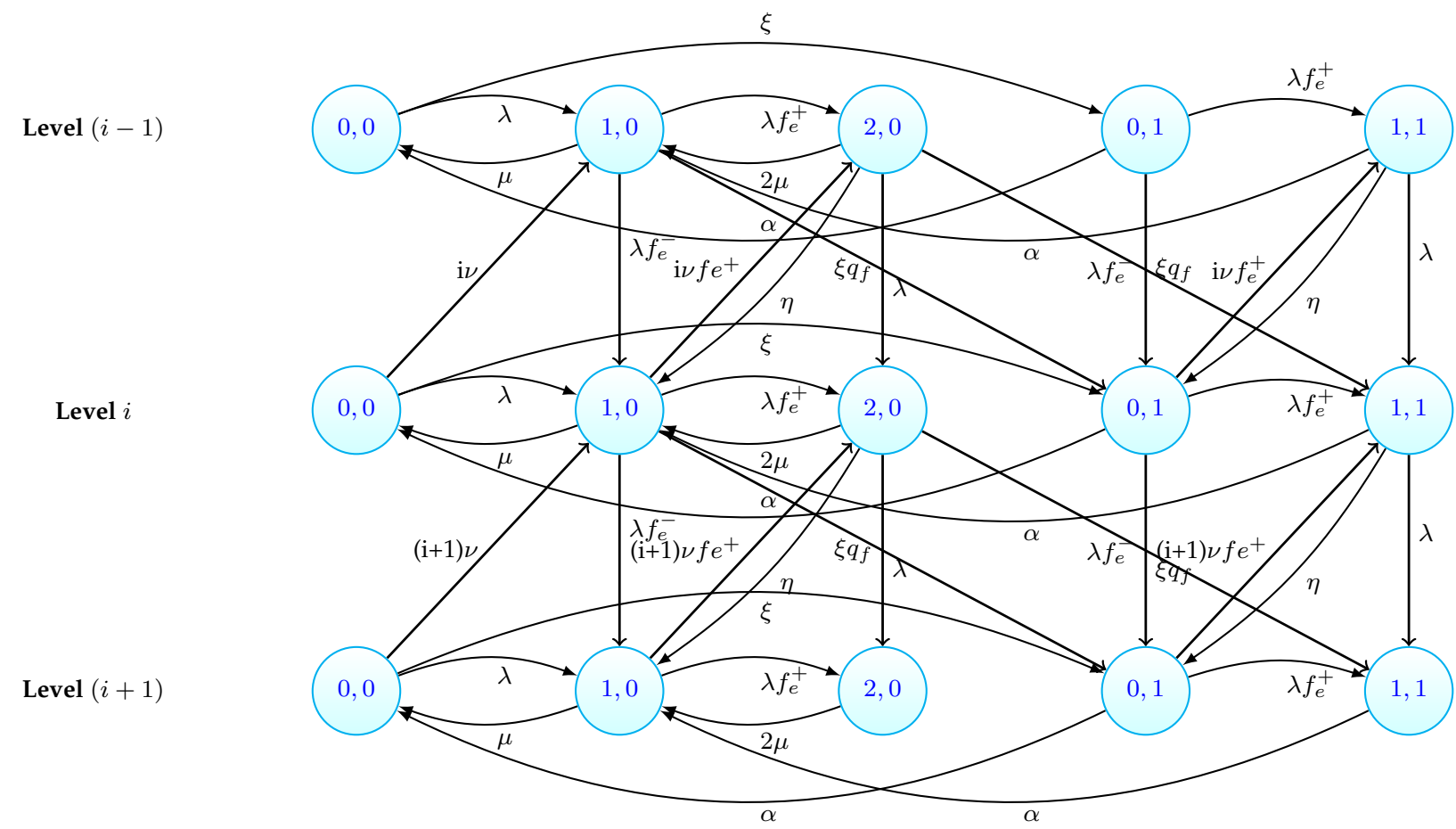

Figure 1. State transition diagram of infinite -source retial queue for $s=1$ (unreliable server) and $q=1$ retrial queue .

$$
\begin{gathered}
\left(\lambda f_{e}^{+}+\mu+i \nu f_{e}^{+}+\lambda f_{e}^{-}\right) p(i, 1,0)=\lambda p(i, 0,0)+\mu p(i, 2,0)+\alpha p(i, 1,1)+(i+1) \nu p(i+1,0,0) \\
\left(\mu+\xi q_{f}+\eta+\lambda\right) p(i, 2,0)=\lambda f_{e}^{+} p(i, 1,0)+i \nu f_{e}^{+} p(i+1,1,0)+\lambda p(i-1,2,0) \\
\left(\lambda f_{e}^{+}+\alpha+i \nu f_{e}^{+}+\lambda f_{e}^{-}\right) p(i, 0,1)=\xi p(i, 0,0)+\eta p(i-1,1,1)+\lambda f_{e}^{-} p(i-1,0,1)+\xi q_{f} p(i-1,1,0) \\
(\alpha+\eta+\lambda) p(i, 1,1)=\lambda f_{e}^{+} p(i, 0,1)+(i+1) \nu f_{e}^{+} p(i+1,0,1)+\xi q_{f} p(i-1,2,0)+\lambda p(i-1,1,1) \\
\sum_{i=0}^{\infty}[p(i, 0,0)+p(i, 1,0)+p(i, 2,0)+p(i, 0,1)+p(i, 1,1)]=1
\end{gathered}
$$

Note that is not easy to solve for the state probabilities recursively or by the method of generating functions, for this reason an approximate analysis is instead using a Phase Merging Algorithm developed by Korolyuk [13] and Courtois [8].

\section{Application of the Phase Merging Algorithm}

The objective of the phase-merging algorithm is to approximate the steady-state probability distribution of $\{(N(t), C(t), F(t)): t \geq 0\}$ by approximating the conditional probability distribution of the status of the service area, given the level of the orbit and by approximating the marginal probability distribution of the number of customers in orbit, for more detailed, see Brian [6].

To make use of the algorithm, we proceed as follows: First, partition the state space into individual levels where the index of each level corresponds to the number of customers in the orbit. This step results in an infinite number of classes (or levels) which can be analyzed individually. Denote this as class $A_{i}$, for level $i, i \geq 0$.

Note that each class has an identical structure and is analyzed as a CTMC from which the approximate conditional probabilities are obtained, see Figure 2

For each $i \geq 0$, the transition rates for this process are described in the following generator matrix $Q_{i}$

$$
Q_{i}=\left(\begin{array}{ccccc}
-(\lambda+\xi) & \lambda & 0 & \xi & 0 \\
\mu & -\left(\lambda f_{e}^{+}+\mu\right) & \lambda f_{e}^{+} & 0 & 0 \\
0 & \mu & -\mu & 0 & 0 \\
\alpha & 0 & 0 & -\left(\alpha+\lambda f_{e}^{+}\right) & \lambda f_{e}^{+} \\
0 & \alpha & 0 & 0 & -\alpha .
\end{array}\right)
$$


Level $(i)$

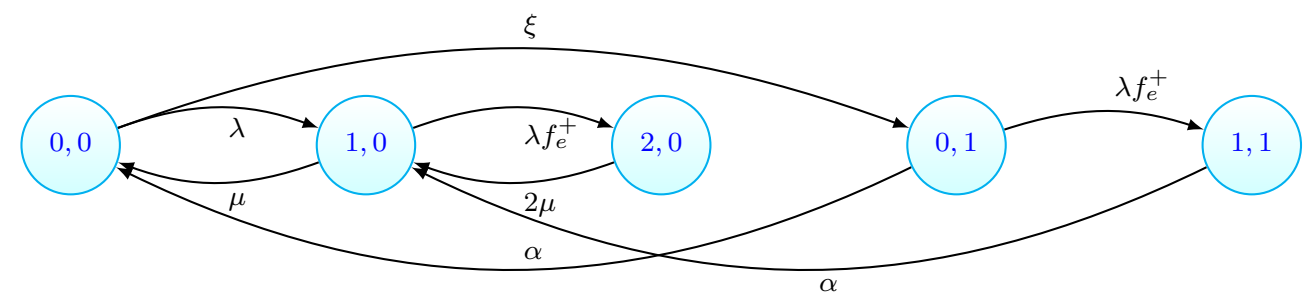

Figure 2. The class $A_{i}$.

The following system yields,

$$
\begin{aligned}
(\lambda+\xi) p_{(0,0) \mid i} & =\mu p_{(1,0) \mid i}+\alpha p_{(1,1) \mid i} \\
\left(\lambda f_{e}^{+}+\mu\right) p_{(1,0) \mid i} & =\lambda p_{(0,0) \mid i}+\mu p_{(2,0) \mid i}+\alpha p_{(1,1) \mid i} \\
\mu p_{(2,0) \mid i} & =\lambda f_{e}^{+} p_{(1,0) \mid i} \\
\left(\lambda f_{e}^{+}+\alpha\right) p_{(0,1) \mid i} & =\xi p_{0,0 \mid i} \\
\alpha p_{(1,1) \mid i} & =\lambda f_{e}^{+} p_{(0,1) \mid i} \\
\sum_{i=0}^{\infty}[p(i, 0,0)+p(i, 1,0)+p(i, 2,0)+p(i, 0,1)+p(i, 1,1)] & =1 .
\end{aligned}
$$

Solving the equations $p_{(j, k) \mid i} Q_{i}=0,(j \in 0,1,2 ; k \in 0,1)$ and $p_{i} e=1$ (where $e$ is a column vector of ones), the conditional probabilities are obtained for all $i \geq 0$, using the Maple Calculation Software

$$
\begin{aligned}
& p_{(0,0) / i}=D^{-1}\left(2 \mu^{2} \alpha^{2}+2 \mu^{2} \alpha \lambda f_{e}^{+}\right) \\
& p_{(1,0) / i}=D^{-1}\left(\mu \alpha(2 \lambda+\xi)\left(\alpha+\lambda f_{e}^{+}\right)\right) \\
& p_{(2,0) / i}=D^{-1}\left(\lambda f_{e}^{+} \alpha(2 \lambda+\xi)\left(\alpha+\lambda f_{e}^{+}\right)\right) \\
& p_{(0,1) / i}=D^{-1}\left(2 \alpha \mu^{2} \xi\right) \\
& p_{(1,1) / i}=D^{-1}\left(\mu^{2} \xi\left(\alpha+\lambda f_{e}^{+}\right)\right) .
\end{aligned}
$$

The constant $D$ is given by the term

$$
\begin{aligned}
D= & 3 \mu^{2} \alpha \xi+\mu^{2} \xi \lambda f_{e}^{+}+2 \mu^{2} \alpha^{2}+2 \mu^{2} \alpha \lambda f_{e}^{+}+\mu \alpha^{2} \xi+\mu \alpha \xi \lambda f_{e}^{+}+2 \alpha^{2} \lambda \mu+2 \mu \alpha \lambda^{2} f_{e}^{+}+\alpha^{2} \xi \lambda f_{e}^{+} \\
& +\alpha \xi \lambda^{2}\left(f_{e}^{+}\right)^{2}+2 \lambda^{2} \alpha^{2} f_{e}^{+}+2 \alpha \lambda^{3}\left(f_{e}^{+}\right)^{2} .
\end{aligned}
$$

Next, we merge all states within the class $A_{i}$ into one state corresponding to the level of orbit, $i$.

The infinitesimal generator, of the merged model is

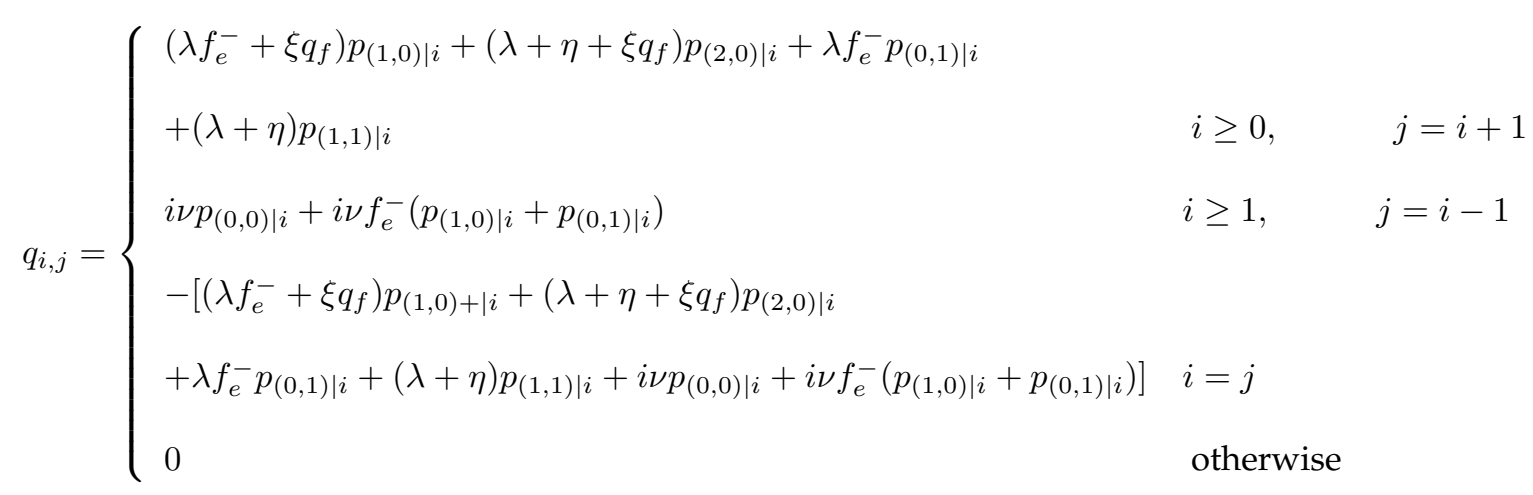


Using the substitutions, $\hat{\lambda}=\left(\lambda f_{e}^{-}+\xi q_{f}\right) p_{(1,0) \mid i}+\left(\lambda+\eta+\xi q_{f}\right) p_{(2,0) \mid i}+\lambda f_{e}^{-} p_{(0,1) \mid i}+(\lambda+\eta) p_{(1,1) \mid i}$ and $\hat{\nu}=\nu p_{(0,0) \mid i}+\nu f_{e}^{-}\left(p_{(1,0) \mid i}+p_{(0,1) \mid i}\right)$, our infinitesimal generator, $q_{i, j}$, of the merged model become

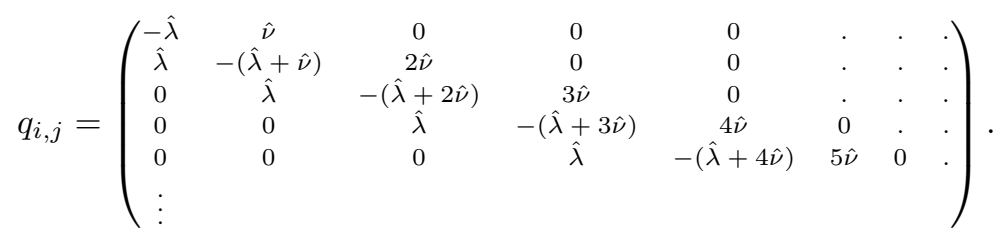

We see that the analysis of this system is analogous to the $M / M / \infty$ queueing system. Using the method of arc cuts, we recursively solve for the steady-state probability vector, $\pi=\left[\pi_{0}, \pi_{1}, \pi_{2}, \ldots\right]$ solving recursively the system equations, we obtain

$$
\begin{aligned}
& \hat{\lambda} \pi_{0}=\hat{\nu} \pi_{1} \Rightarrow \pi_{1}=\frac{\hat{\lambda}}{\hat{\nu}} \pi_{0}, \\
& \hat{\lambda} \pi_{1}=2 \hat{\nu} \pi_{2} \Rightarrow \pi_{2}=\frac{1}{2}\left(\frac{\hat{\lambda}}{\hat{\nu}}\right)^{2} \pi_{0} .
\end{aligned}
$$

Continuing, it can be shown that

$$
\pi_{i}=\frac{1}{i !}\left(\frac{\hat{\lambda}}{\hat{\nu}}\right)^{i} \pi_{0}, \quad i \geq 0
$$

Using the normalization equation, $\sum_{j=0}^{\infty} \pi_{j}=1$, the solution for $\pi_{0}$ is obtained by

$$
\begin{gathered}
\pi_{0}+\frac{\hat{\lambda}}{\hat{\nu}} \pi_{0}+\frac{1}{2}\left(\frac{\hat{\lambda}}{\hat{\nu}}\right)^{2} \pi_{0}+\frac{1}{6}\left(\frac{\hat{\lambda}}{\hat{\nu}}\right)^{3} \pi_{0}+\ldots=1 \\
\pi_{0}\left(1+\frac{\hat{\lambda}}{\hat{\nu}}+\frac{1}{2}\left(\frac{\hat{\lambda}}{\hat{\nu}}\right)^{2}+\frac{1}{6}\left(\frac{\hat{\lambda}}{\hat{\nu}}\right)^{3} \pi_{0}+\ldots\right)=1 \\
\pi_{0}\left(\sum_{j=0}^{\infty} \frac{1}{j !}\left(\frac{\hat{\lambda}}{\hat{\nu}}\right)^{j}\right)=1 .
\end{gathered}
$$

The infinite series of (4.2) is the Maclaurin power series expansion for $e^{\hat{\lambda} / \hat{\nu}}$. Thus, we see that

$$
\pi_{0}=e^{-\hat{\lambda} / \hat{\nu}}
$$

Substituting $\pi_{0}$ into Equation (4.1) we have the following expression

$$
\pi_{i}=\frac{1}{i !}\left(\frac{\hat{\lambda}}{\hat{\nu}}\right)^{i} e^{-\hat{\lambda} / \hat{\nu}}, \quad i \geq 0
$$

which is the probability mass function for a Poisson distributed random variable with rate parameter $\frac{\hat{\lambda}}{\hat{\nu}}$.

Finally, we approximate the steady-state distribution of $\{(N(t), C(t), F(t)): t \geq 0\}$ by

$$
\begin{aligned}
p(i, j, k) & =p_{(j, k) \mid i} \times \pi_{i} \\
& =\frac{p_{(j, k) \mid i}}{i !}\left(\frac{\hat{\lambda}}{\hat{\nu}}\right)^{i} e^{-\hat{\lambda} / \hat{\nu}} \quad i \geq 0 ; j \in\{0,1,2\} ; k \in\{0,1\} .
\end{aligned}
$$

\section{Results}

Using this joint distribution, we can approximate the standard queueing performance measures.

\section{Mean Orbit Length}

$$
\begin{aligned}
E[N] & \approx \frac{\hat{\lambda}}{\hat{\hat{v}}} \\
& =\frac{\left(\lambda f_{e}^{-}+\xi q_{f}\right) p_{(1,0)+\mid i}+\left(\lambda+\eta+\xi q_{f}\right) p_{(2,0) \mid i}+\lambda f_{e}^{-} p_{(0,1) \mid i}+(\lambda+\eta) p_{(1,1) \mid i}}{\nu p_{(0,0)}+\nu f_{e}^{-}\left(p_{(1,0) \mid i}+p_{(0,1) \mid i}\right)} .
\end{aligned}
$$




\section{Mean Number of Customer in Service Area}

The approximate expression for the expected number of customer at the Service Area is given by:

$$
E[A]=\sum_{i=0}^{\infty}[p(i, 1,0)+p(i, 2,0)+p(i, 1,1)] .
$$

\section{Mean Queue Length}

The mean queue length $M Q L$ is given by :

$$
M Q L=\sum_{i=0}^{\infty}[p(i, 1,1)+p(i, 2,0)] .
$$

\section{Model Generalization}

In section 3, the investigation was restricted for $q=1$ and $s=1$ to show the principles, in this section we apply the Algorithm for a higher values of $s$ and $q$ and to characterize the status of the service area at any time, we need a set of $\sum_{n=1}^{s} n+(s+1)(q+1)$ pairs of $(j, k)$. Recall that all the random times are exponentially distributed and the stochastic process is a continuous-time Markov chain (CTMC) on the state space $A=\{(i, j, k): i \geq 0, j \in$ $\{0,1,2, \ldots, q+s\} ; k \in\{0,1,2, \ldots, s\}\}$.

We assume that as $t \rightarrow \infty$, the steady-state distribution of $\{(N(t), C(t), F(t)): t \geq 0\}$ exists and we need the sufficient condition for ergodicity, see Falin [12]

$$
\frac{\lambda}{s \mu}\left(1+\frac{\xi}{\alpha}+\eta\right)<1
$$

Define $p(i, j, k)$ as the limiting probability that the system is in the state $(i, j, k)$ where $(i, j, k) \in A$. Defined mathematically

$$
p(i, j, k)=\lim _{t \rightarrow \infty} P(N(t)=i, C(t)=j, F(t)=k) .
$$

First, we reduce the dimensionality of the state space by defining $X(t)$ as the status of the service area at time $t$ (outlined in Table 1 ), such that $X(t) \in\left\{1,2,3, \ldots, \sum_{n=1}^{S} n+(s+1)(q+1)\right\}$.

\begin{tabular}{cc}
\hline State $(j, k)$ & Index(l) \\
\hline$(0,0)$ & 1 \\
$(1,0)$ & 2 \\
$(2,0)$ & 3 \\
$\vdots$ & $\vdots$ \\
$(s, 0)$ & $s+1$ \\
$(s+1,0)$ & $s+2$ \\
$\vdots$ & $\vdots$ \\
$(c, 0)$ & $\mathrm{c}+1$ \\
$(0,1)$ & $\mathrm{c}+2$ \\
$(1,1)$ & $\mathrm{c}+3$ \\
$\vdots$ & $\vdots$ \\
$(q, s)$ & $\sum_{n=1}^{s} n+(s+1)(q+1)$ \\
\hline
\end{tabular}

Table 1 : Substitution for service area status.

Since the number of customers in the orbit can theoretically reach infinity, the state space of the system can be partitioned into a countable number of classes. As noted previously, the state space $A$ is partitioned as the countable union,

$$
A=\bigcup_{i=0}^{\infty} A_{i}, \quad A_{i} \cap A_{j}=\emptyset, \quad i \neq j
$$


where $A_{i}=\left\{(i, l): l=1,2, \ldots, \sum_{n=1}^{s} n+(s+1)(q+1)\right\}, i \geq 0$. each class is identical in structure so that only one class needs to be analyzed.

We denote $p_{l \mid i}$ as the limiting conditional probability of the service area being in state $l$ given that there are $i$ customers in orbit

$$
p_{l \mid i}=\lim _{t \rightarrow \infty} P(X(t)=l / N(t)=i), l=1,2, \ldots, \sum_{n=1}^{s} n+(s+1)(q+1)
$$

For each $i \geq 0$, the transition rates for this process are described in the generator matrix $Q_{i}$.

$$
\begin{aligned}
& (\lambda+s \xi) p_{(0,0) \mid i} \quad=\mu p_{(1,0) \mid i}+\alpha p_{(0,1) \mid i} \\
& (\lambda+(s-1) \xi+\mu) p_{(1,0) \mid i} \quad=\lambda p_{(0,0) \mid i}+2 \mu p_{(2,0) \mid i}+\alpha p_{(1,1) \mid i} \\
& (\lambda+(s-2) \xi+2 \mu) p_{(2,0) \mid i} \quad=\quad \lambda p_{(1,0) \mid i}+3 \mu p_{(3,0) \mid i}+\alpha p_{(2,1) \mid i} \\
& \vdots \quad \vdots \\
& (\lambda+\xi+(s-1) \mu) p_{(s-1,0) \mid i} \quad=\lambda p_{(s-2,0) \mid i}+s \mu p_{(s, 0) \mid i}+\alpha p_{(s-1,1) \mid i} \\
& \left(\lambda f_{e}^{+}+s \mu\right) p_{(s, 0) \mid i} \quad=\lambda p_{(s-1,0) \mid i}+s \mu p_{(s+1,0) \mid i}+\alpha p_{(s, 1) \mid i} \\
& \left(\lambda f_{e}^{+}+s \mu\right) p_{(j, 0) \mid i} \quad=\lambda f_{e}^{+} p_{(j-1,0) \mid i}+s \mu p_{(j+1,0) \mid i}+\alpha p_{(j, 1) \mid i} \text { with } j \in\{s+1, s+2, \ldots s+q-1\} \\
& \sup _{(s+q, 0) \mid i} \quad=\lambda f_{e}^{+} p_{(s+q-1,0) \mid i} \\
& (\lambda+(s-(j+k)) \xi+j \mu+k \alpha) p_{(j, k) \mid i}=(j+1) \mu p_{(j+1, k) \mid i}+(k+1) \alpha p_{(j, k+1) \mid i}+\lambda p_{(j-1, k) \mid i} \text { with } j+k<S ; j \neq 0 ; k \neq 0 \\
& \left(\lambda f_{e}^{+}+(s-k) \mu+k \alpha\right) p_{(j, k) \mid i}=\lambda f_{e}^{+} p_{(j-1, k) \mid i}+(s-k) \mu p_{(j+1, k) \mid i}+(k+1) \alpha p_{(j, k+1) \mid i} \text { with } s<j+k<C ; k \neq 0, s \\
& \left(\lambda f_{e}^{+}+(s-k) \mu+k \alpha\right) p_{(j, k) \mid i}=\lambda p_{(j-1, k) \mid i}+(s-k) \mu p_{(j+1, k) \mid i}+(k+1) \alpha p_{(j, k+1) \mid i}+\xi p_{(j, k-1) \mid i} \text { with } j+k=s \\
& ((s-k) \mu+k \alpha) p_{j, k \mid i} \quad=\lambda f_{e}^{+} p_{(j-1, k) \mid i} \text { with } j+k=s ; k \neq 0 ; k \neq s \\
& (\lambda+(s-1) \xi+\alpha) p_{(0,1) \mid i}=s \xi p_{(0,0) \mid i}+\mu p_{(1,1) \mid i}+2 \alpha p_{(0,2) \mid i} \\
& (\lambda+(s-2) \xi+2 \alpha) p_{(0,2) \mid i}=(s-1) \xi p_{0,1 \mid i}+\mu p_{(1,2) \mid i}+3 \alpha p_{(0,3) \mid i} \\
& (\lambda+(s-3) \xi+3 \alpha) p_{(0,3) \mid i} \quad=(s-2) \xi p_{0,2 \mid i}+\mu p_{(1,3) \mid i}+4 \alpha p_{(0,4) \mid i} \\
& (\lambda+(s-1) \alpha+\xi) p_{(0, s-1) \mid i} \quad=2 \xi p_{0, s-2 \mid i}+\mu p_{(1, s-1) \mid i}+s \alpha p_{(0, s) \mid i} \\
& \left(\lambda f_{e}^{+}+s \alpha\right) p_{(0, s) \mid i} \quad=\xi p_{0, s-1 \mid i} \\
& \left(\lambda f_{e}^{+}+s \alpha\right) p_{(j, s) \mid i} \quad=\lambda f_{e}^{+} p_{(j-1, s) \mid i} \quad \text { with } j \in 1,2, \ldots q-1 \\
& \operatorname{sop}_{(q, s) \mid i} \quad=\lambda f_{e}^{+} p_{(q-1, s) \mid i} \\
& \sum_{n=1}^{s} n+(s+1)(q+1) \\
& \sum_{l=1} p_{l \mid i} \quad=1
\end{aligned}
$$

Aggregating the states of each class $A_{i}$. The rates of transitions between the "macro-states" are expressed in the infinitesimal generator matrix with elements,

$$
q_{i, j}= \begin{cases}\sum_{j+k=c} \lambda p_{(j, k) \mid i}+\sum_{\substack{j+k \leq c \\ j>c}} q \eta p_{(j, k) \mid i}+\sum_{\substack{j+k<c \\ j>s}} \lambda f_{e}^{-} p_{(j, k) \mid i}+\sum_{\substack{j+k \leq c \\ j \neq 0 \\ k<s}} j \xi q_{f} p_{(j, k) \mid i} & i \geq 0, j=i+1 \\ i\left[\nu \sum_{j+k \leq s} p_{(j, k) \mid i}+\nu f_{e}^{+} \sum_{s<j+k \neq c} p_{(j, k) \mid i}\right] & i \geq 1, \quad j=i-1 \\ -\left[\sum_{j+k=c}^{j+k \leq s} \lambda p_{(j, k) \mid i}+\sum_{\substack{j+k \leq c \\ j>c}} q \eta p_{(j, k) \mid i}+\sum_{\substack{j+k<c \\ j>s}} \lambda f_{e}^{-} p_{(j, k) \mid i}+\sum_{\substack{j+k \leq c \\ j \neq 0}} j \xi q_{f} p_{(j, k) \mid i}\right. & \\ \left.+i \nu \sum_{j<s \leq s} p_{(j, k) \mid i}+i \nu f_{e}^{+} \sum_{s<j+k \neq c} p_{(j, k) \mid i}\right] & i=j \\ 0 & \text { otherwise . }\end{cases}
$$

We use the following substitutions for $i, i \geq 0$

$$
\begin{aligned}
\hat{\lambda} & =\sum_{j+k=c} \lambda p_{(j, k) \mid i}+\sum_{\substack{j+k \leq c \\
j>c}} q \eta p_{(j, k) \mid i}+\sum_{\substack{j+k<c \\
j>S}} \lambda f_{e}^{-} p_{(j, k) \mid i}+\sum_{\substack{j+k \leq c \\
j \neq 0 \\
j<s}} j \xi q_{f} p_{(j, k) \mid i} \\
\hat{\nu} & =\nu \sum_{j+k \leq s} p_{(j, k) \mid i}+\nu f_{e}^{+} \sum_{s<j+k \neq c} p_{(j, k) \mid i} .
\end{aligned}
$$




\section{- Mean Orbit Length}

For the infinite source multi service retrial queue, the steady-state distribution will be also Poisson with parameter $\hat{\lambda} / \hat{\nu}$. Denoting $N$ as the steady-state number of customers in orbit when the mean orbit size is approximated by

$$
E[N] \approx \frac{\sum_{j+k=c} \lambda p_{(j, k) \mid i}+\sum_{\substack{j+k \leq c \\ j>c}} q \eta p_{(j, k) \mid i}+\sum_{\substack{j+k<c \\ j>s}} \lambda f_{e}^{-} p_{(j, k) \mid i}+\sum_{\substack{j+k \leq c \\ j \neq 0 \\ k<s}} j \xi q_{f} p_{(j, k) \mid i}}{\nu \sum_{j+k \leq s} p_{(j, k) \mid i}+\nu f_{e}^{+} \sum_{s<j+k \neq c} p_{(j, k) \mid i}} .
$$

- Mean Number of customers in Service

Denoting $N_{e}$ as the random number of customers at the servers, the approximate expression for the expected number of customers at the servers can be computed using the approximate steady-state joint probabilities derived in the last step of the algorithm and it is given by

$$
E\left[N_{e}\right]=\sum_{i=0}^{\infty} \sum_{\substack{j \neq 0 \\ k \neq s}} p(i, j, k)
$$

\section{- Mean Number of customers in Service Area}

Denoting $N_{r}$ as the random number of customers at the service area

$$
E\left[N_{r}\right]=E\left[N_{e}\right]+\sum_{i=0}^{\infty} \sum_{\substack{j \neq 0 \\ k=s}} p(i, j, k)
$$

\section{- Mean Queue Length}

The mean queue length $M Q L$ is given by :

$$
M Q L=\sum_{i=0}^{\infty}\left(\sum_{j+k=s+1}^{c} p(i, j, k)\right) .
$$

\section{- Steady-State System Size and Sojourn Time}

Denoting $L$ as the steady-state number of customers in the system, to calculate it, we simply sum the expressions for $E[N]$ and $E\left[N_{r}\right]$.

The steady-state mean sojourn time, $W$, follows directly from Little's law.

$$
\begin{aligned}
& L \approx E[N]+E\left[N_{r}\right] \\
& W \approx \frac{L}{\lambda} .
\end{aligned}
$$

\section{- The probability mass function of number of busy servers}

$$
P(\text { all S servers are busy })=\sum_{i=0}^{\infty} \sum_{j=s}^{c} p(i, j, 0) .
$$

\section{- The probability mass function of number of servers in breakdown}

$$
P(\text { all S servers are in breakdown })=\sum_{i=0}^{\infty} \sum_{q=0}^{c-S} p(i, q, s) .
$$




\section{Numerical Results}

In this section, we will give some numerical results to illustrate how the various parameters of the model influence on the system performances measures for $s=1$ and $q=1$.

Table 2: Retrial rate $(\nu)$ and impatience rate $(\eta)$ against mean number of customers in the orbit for $\lambda=0.5$, $\mu=2, \alpha=0.5, \xi=0.05, f_{e}^{-}=f_{e}^{+}=0.5$ and $q_{f}=0.5$.

\begin{tabular}{|c|c|c|c|c|c|c|c|c|c|c|}
\hline$\nu$ & $\eta=0.02$ & $\eta=0.04$ & $\eta=0.06$ & $\eta=0.08$ & $\eta=0.1$ & $\eta=0.2$ & $\eta=0.4$ & $\eta=0.6$ & $\eta=0.8$ & $\eta=1$ \\
\hline 0.02 & 5.698 & 5.770 & 5.841 & 5.912 & 5.983 & 6.338 & 7.050 & 7.761 & 8.472 & 9.183 \\
0.04 & 2.849 & 2.885 & 2.920 & 2.956 & 2.991 & 3.169 & 3.524 & 3.880 & 4.236 & 4.591 \\
0.06 & 1.899 & 1.923 & 1.947 & 1.970 & 1.994 & 2.113 & 2.350 & 2.587 & 2.824 & 3.061 \\
0.08 & 1.424 & 1.442 & 1.460 & 1.478 & 1.495 & 1.584 & 1.762 & 1.940 & 2.118 & 2.295 \\
0.1 & 1.139 & 1.154 & 1.168 & 1.182 & 1.196 & 1.267 & 1.410 & 1.552 & 1.694 & 1.836 \\
0.2 & 0.569 & 0.577 & 0.584 & 0.591 & 0.598 & 0.633 & 0.705 & 0.776 & 0.847 & 0.918 \\
0.4 & 0.284 & 0.288 & 0.292 & 0.295 & 0.299 & 0.317 & 0.352 & 0.388 & 0.423 & 0.459 \\
0.6 & 0.189 & 0.192 & 0.194 & 0.197 & 0.199 & 0.211 & 0.235 & 0.258 & 0.282 & 0.306 \\
0.8 & 0.142 & 0.144 & 0.146 & 0.147 & 0.149 & 0.158 & 0.176 & 0.194 & 0.211 & 0.229 \\
1 & 0.114 & 0.115 & 0.116 & 0.118 & 0.119 & 0.126 & 0.140 & 0.155 & 0.169 & 0.183 \\
\hline
\end{tabular}

\begin{tabular}{|c|c|c|c|c|}
\hline$p_{(0,0) \mid i}$ & $p_{(1,0) \mid i}$ & $p_{(2,0) \mid i}$ & $p_{(0,1) \mid i}$ & $p_{(1,1) \mid i}$ \\
\hline 0.708 & 0.185 & 0.023 & 0.047 & 0.035 \\
\hline
\end{tabular}

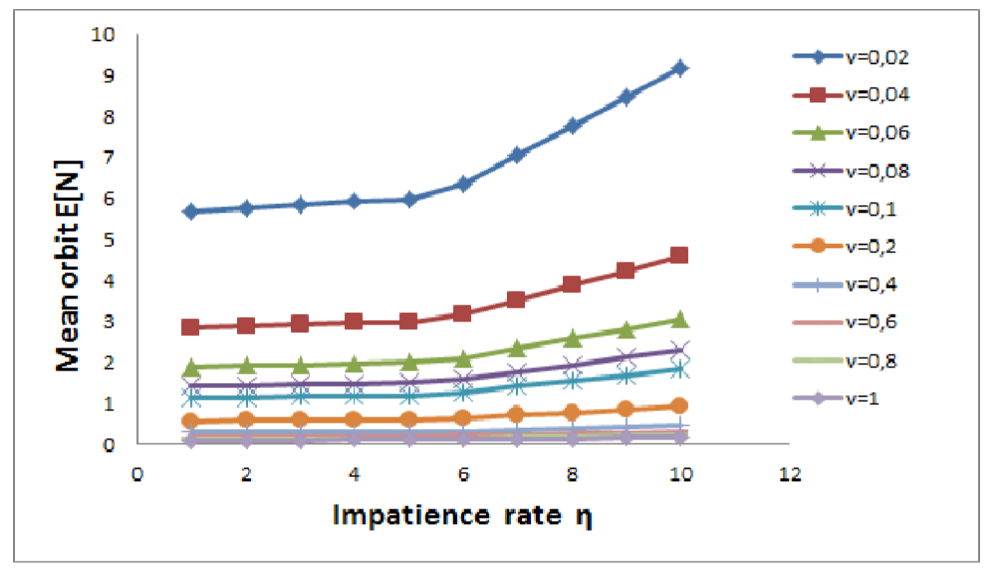

Figure 3

From Figure 3, it can be seen that the mean number of customers in Orbit decreases rapidly as $\nu$ and $\eta$ become higher and exceeds 0.1 .

Table 3: Retrial rate $\nu$ and impatience rate $\eta$ and probability $f_{e}^{+}$against mean number of customers in the orbit for $\lambda=0.5, \mu=2, \alpha=0.5, \xi=0.05$ and $q_{f}=0.5$.

\begin{tabular}{|c|c|c|c|c|c|c|}
\hline$\nu=\eta$ & $f_{e}^{+}=0$ & $f_{e}^{+}=0.2$ & $f_{e}^{+}=0.4$ & $f_{e}^{+}=0.6$ & $f_{e}^{+}=0.8$ & $f_{e}^{+}=1$ \\
\hline 0.02 & 17.581 & 13.866 & 11.112 & 8.963 & 7.225 & 5.783 \\
0.04 & 8.815 & 6.978 & 5.618 & 4.558 & 3.701 & 2.991 \\
0.06 & 5.893 & 4.683 & 3.787 & 3.090 & 2.527 & 2.060 \\
0.08 & 4.432 & 3.535 & 2.872 & 2.355 & 1.939 & 1.594 \\
0.1 & 3.556 & 2.846 & 2.322 & 1.915 & 1.587 & 1.315 \\
0.3 & 1.218 & 1.009 & 0.857 & 0.740 & 0.647 & 0.570 \\
0.5 & 0.751 & 0.642 & 0.564 & 0.505 & 0.459 & 0.421 \\
0.7 & 0.550 & 0.484 & 0.439 & 0.405 & 0.378 & 0.357 \\
\hline
\end{tabular}

From Table 3, it is also interested in the sensitivity of the approximation procedure to vary $f_{e}^{+}$from 0 to 1 in increments of 0.2 and provide the results for the same values of $\lambda, \mu, \alpha, \xi$ of Table 1 and it can be shown that along 
the increases of $\nu$ and $\eta$ for the same values, the mean number of customers in orbit decreases also when the values of $f_{e}^{+}$become larger, this is because there is a high probability $\left(p_{(0,0) \mid i}=0.708\right)$ that the customer find the server idle when he retry to enter the server again for small value of $\lambda$.

Table 4: Retrial rate $\nu$, impatience rate $\eta$ and $\lambda$ against mean number of customers in the orbit for $\mu=15$, $\alpha=0.5, \xi=0.05, f_{e}^{-}=f_{e}^{+}=0.5$ and $q_{f}=0.5$.

\begin{tabular}{|c|c|c|c|c|c|c|}
\hline$\lambda$ & $\nu=\eta=0.5$ & $\nu=\eta=0.6$ & $\nu=\eta=0.7$ & $\nu=\eta=0.8$ & $\nu=\eta=0.9$ & $\nu=\eta=1$ \\
\hline 7 & 4.662 & 3.907 & 3.367 & 2.962 & 2.647 & 2.395 \\
6 & 3.488 & 2.925 & 2.522 & 2.220 & 1.986 & 1.798 \\
5 & 2.502 & 2.100 & 1.812 & 1.597 & 1.430 & 1.296 \\
4 & 1.696 & 1.425 & 1.232 & 1.088 & 0.975 & 0.885 \\
3 & 1.061 & 0.895 & 0.776 & 0.687 & 0.617 & 0.562 \\
2 & 0.589 & 0.500 & 0.436 & 0.388 & 0.351 & 0.321 \\
1 & 0.263 & 0.227 & 0.202 & 0.182 & 0.168 & 0.156 \\
\hline
\end{tabular}

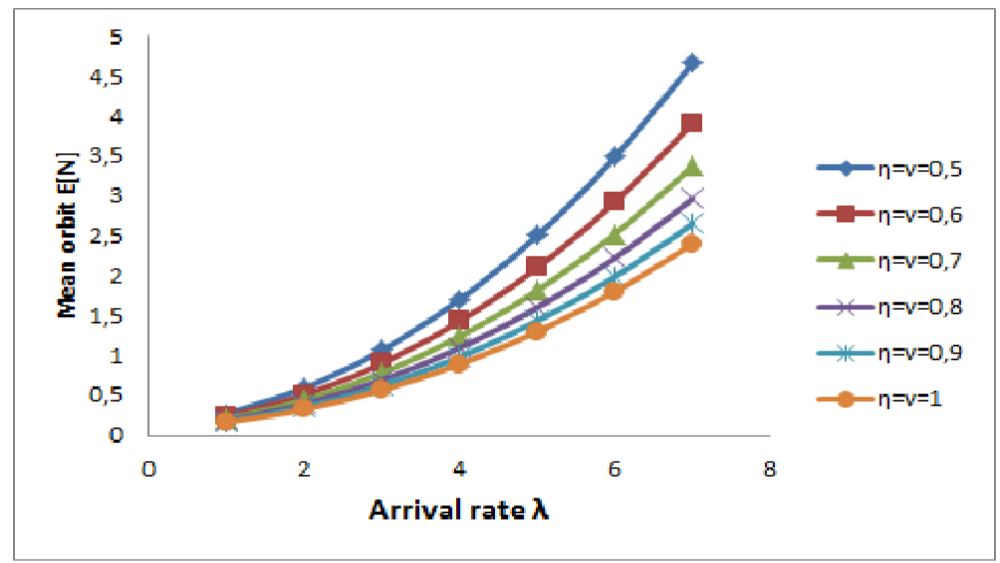

Figure 4

From Figure 4, it is shown that the mean number of customers in orbit increases and the server keep busy for more time as $\lambda$ increase. High generation rate lead to high server utilization.

Table 5: Retrial rate $\xi$ and repair rate $\alpha$ against mean number of customers in the orbit for $\lambda=0.5, \mu=2$, $\nu=0.5, \eta=0.5, f_{e}^{-}=f_{e}^{+}=0.5$ and $q_{f}=0.5$.

\begin{tabular}{|c|c|c|c|c|c|c|c|}
\hline$\xi$ & $\alpha=2$ & $\alpha=2.5$ & $\alpha=3$ & $\alpha=3.5$ & $\alpha=4$ & $\alpha=4.5$ & $\alpha=5$ \\
\hline 0.02 & 0.186 & 0.184 & 0.182 & 0.181 & 0.180 & 0.180 & 0.179 \\
0.04 & 0.206 & 0.202 & 0.199 & 0.196 & 0.195 & 0.193 & 0.192 \\
0.06 & 0.226 & 0.220 & 0.215 & 0.212 & 0.209 & 0.207 & 0.205 \\
0.08 & 0.246 & 0.237 & 0.231 & 0.227 & 0.223 & 0.221 & 0.219 \\
0.1 & 0.266 & 0.255 & 0.247 & 0.242 & 0.238 & 0.235 & 0.232 \\
0.3 & 0.463 & 0.433 & 0.413 & 0.398 & 0.387 & 0.378 & 0.371 \\
0.5 & 0.657 & 0.612 & 0.581 & 0.559 & 0.542 & 0.529 & 0.518 \\
0.7 & 0.847 & 0.791 & 0.753 & 0.725 & 0.703 & 0.686 & 0.672 \\
0.9 & 1.034 & 0.971 & 0.927 & 0.894 & 0.869 & 0.849 & 0.833 \\
1.1 & 1.219 & 1.151 & 1.103 & 1.067 & 1.040 & 1.018 & 1.000 \\
1.3 & 1.402 & 1.331 & 1.281 & 1.243 & 1.214 & 1.192 & 1.173 \\
1.5 & 1.583 & 1.511 & 1.460 & 1.422 & 1.393 & 1.370 & 1.351 \\
\hline
\end{tabular}

From Figure 5, it can be seen that as the Retrial rate $\xi$ increase as the probability that the server is idle decrease and the orbit size increase.

Table 6: Retrial rate $\xi$ and probability $q_{f}$ against mean number of customers in the orbit for $\lambda=0.5, \mu=2$, $\alpha=2, \nu=0.5, \eta=0.5 f_{e}^{-}=f_{e}^{+}=0.5$. 


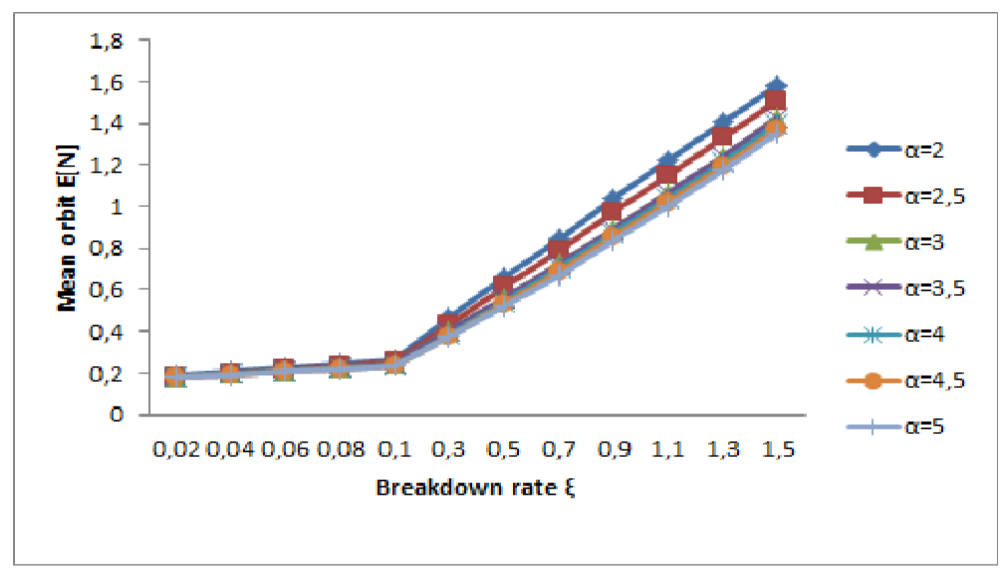

Figure 5

\begin{tabular}{|c|c|c|c|c|c|}
\hline$q_{f}$ & $\xi=0.2$ & $\xi=0.4$ & $\xi=0.6$ & $\xi=0.8$ & $\xi=1$ \\
\hline 0 & 0.309 & 0.436 & 0.550 & 0.652 & 0.745 \\
0.1 & 0.320 & 0.461 & 0.590 & 0.710 & 0.821 \\
0.2 & 0.331 & 0.486 & 0.631 & 0.768 & 0.898 \\
0.3 & 0.343 & 0.511 & 0.671 & 0.825 & 0.974 \\
0.4 & 0.354 & 0.535 & 0.712 & 0.883 & 1.050 \\
0.5 & 0.365 & 0.560 & 0.752 & 0.941 & 1.127 \\
0.6 & 0.377 & 0.585 & 0.793 & 0.999 & 1.203 \\
0.7 & 0.388 & 0.610 & 0.833 & 1.056 & 1.280 \\
0.8 & 0.399 & 0.635 & 0.874 & 1.114 & 1.356 \\
0.9 & 0.411 & 0.660 & 0.914 & 1.172 & 1.433 \\
1 & 0.422 & 0.685 & 0.955 & 1.229 & 1.509 \\
\hline
\end{tabular}

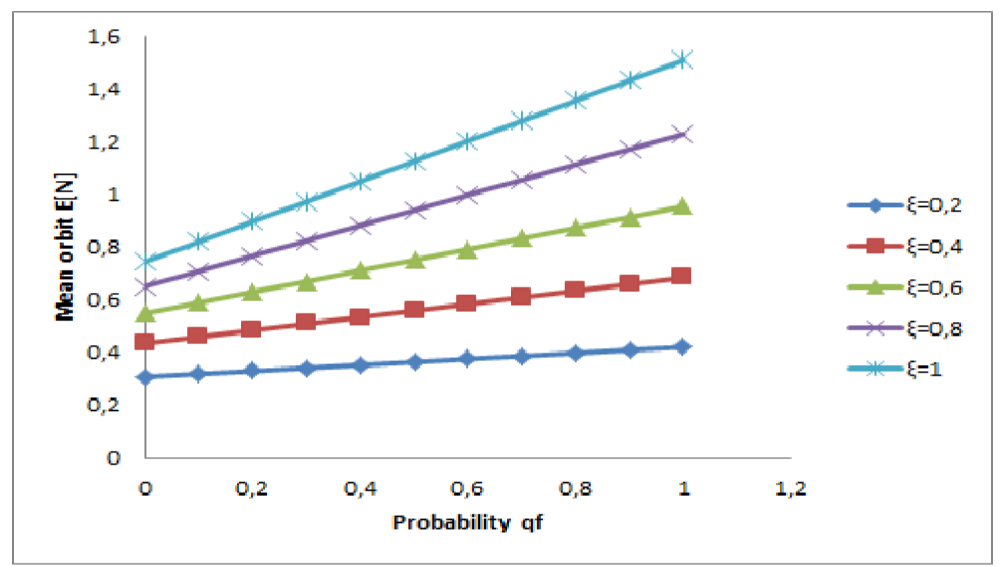

Figure 6

From Figure 6, it can be seen that the orbit size length is not affected remarkably by the probability $q_{f}$ when the breakdown rate $\xi$ is less than 0.2 .

\section{Conclusion}

- The means number of busy and breakdown servers is independent of retrial rate $\nu$ and impatient rate $\eta$.

- The orbit size is not affected remarkably by the increase of the breakdown rate $\xi$ when $\lambda$ is small. 


\section{References}

[1] Aguir, S., Karaesmen, F., Aksin, O.Z., Chauvet,F., The impact of retrials on call center performance, OR Spectr. 26 (2004) 353-376.

[2] Artalejo, J.R., A classified bibliography of research on retrial queues: Progress in 1990-1999.,Busin. Econ.,7 (1999), 187-211.

[3] Artalejo, J.R., Accessible bibliography on retrial queues, Math. Comp. Mod., 30 (1999), 1-6.

[4] Artalejo, J.R. and Gomez-Corral, A., Retrial Queueing Systems: A Computational Approach, Springer, Spain, pp: 318. (2008)

[5] Artalejo, J.R., Accessible bibliography on retrial queues: Progress in 2000-2009., Math. Comp. Mod., 51 (2010), 1071-1081.

[6] Brian, Crawford, P., Approximate analysis of an unreliable M/M/2 retrial queue, thesis, (2007)

[7] Cohen, J. W., Basic problems of telephone traffic theory and the influence of repeated calls, Philips Telecommunication Review, 18, No. 2, 1957.

[8] Courtois, P.J., Decomposability, instabilities, and saturation in multiprogramming systems, Communications of the ACM, 18 (7) (1975), 371-377.

[9] Elhaddad, M., Belarbi, F., Approximate analysis of an unreliable $M / M / c$ retrial queue with phase merging algorithm, New Trends in Mathematical Sciences, 4, (2016), 9-21.

[10] Falin, G., A survey of retrial queues, Queueing Sys., 7, (1990), 127-167.

[11] Falin, G., Artalejo, J.R., Approximations for multiserver queues with balking/retrial discipline, Or Spektrum, 17, (1995), 239-244.

[12] Falin, G.I. and Templeton, J.G.C., Retrial queues, Champman and Hall, London, pp: 328. (1997)

[13] Korolyuk, V.S. and Korolyuk, V.V., Stochastic models of systems. Kluwer Academic Publishers, Boston (1999).

[14] Korolyuk, V. S., Melikov, A. Z., Ponomarenko, L. A., Rustamov, A. M., Methods for analysis of multi-channel queueing system with instantaneous and delayed feedbacks,Cybernetics and Systems Analysis, Vol. 52, No. 1 (2016), 58-70.

[15] Kulkarni, V.G. and Choi, B.D., Retrial queues with server subject to breakdown and repairs, Queueing Sys., 7 (1990), 191-208.

[16] Kosten, L., On the influence of repeated calls in the theory of probabilities of blocking,De Ingenieur, 59,(1947)

[17] Shin, Y.W., Choo, T.S., $M / M / s$ queue with impatient customers and retrials, Applied Mathematical Modelling, 33 (2009), 2596-2606.

[18] Subramanian, M.G., Ayyappan, G. and Sekar, G., $M / M / c$ Retrial queueing system with breakdown and repair of services, Asian Journal of Mthematics and Statistics 4 (4) (2011), 214-223.

\section{Affiliations}

MERIEM ELHADDAD

ADDREss: Laboratory Statistics and Stochastic Processes, University Djillali Liabes of Sidi Bel Abbes, P.O.Box 89, Algeria

E-MAIL: meriem.elhaddad61@gmail.com

ORCID ID:0000-0003-1137-203X

FAIZA LIMAM-BELARBI

AdDress: Laboratory Statistics and Stochastic Processes, University Djillali Liabes of Sidi Bel Abbes, P.O.Box 89, 
Algeria
E-MAIL: faiza_belarbi@yahoo.fr
ORCID ID:0000-0002-5424-1614 